\title{
Cultural Competency of Dental Students: Clinical Supervisors' Perceptions
}

\author{
R. Mariño, A. Ghanim, M. Morgan and S. Barrow
}

(1)

Melbourne Dental School, University of Melbourne, Melbourne, Vic., Australia

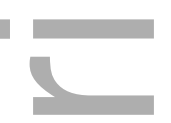

Running title: Clinical supervisors' reflections on students' cultural competency

Correspondence to:

A/Prof Rodrigo C: Oral Health Cooperative Research Centre, Melbourne Dental School, The University of Melbourne, Melbourne, Vic. 3010, Australia.

Tel.: +61 (0)39341 1558

Fax: +61 (0)39341 1597

E-mail: $\underline{\text { r.marino@unimelb.edu.au }}$

Keywords: dental students, cultural competence; cultural diversity; dental education; dental educators

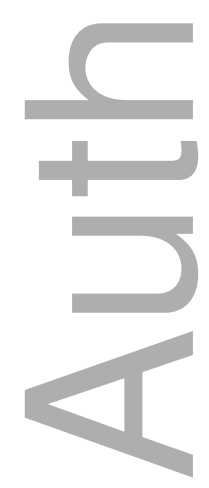

This is the author manuscript accepted for publication and has undergone full peer review but has not been through the copyediting, typesetting, pagination and proofreading process, which may lead to differences between this version and the Version of Record. Please cite this article as doi: $10.1111 /$ eje.12227

This article is protected by copyright. All rights reserved 
Received Date : 15-Feb-2016

Revised Date : 14-May-2016

Accepted Date : 20-Jun-2016

Article type :Original Article

$-$
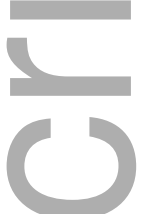

Cultural Competency and Communications Skills of Dental Students: Clinical

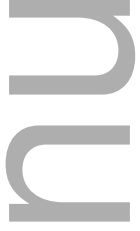
Supervisors' Perceptions

R. Mariño, A. Ghanim, M. Morgan and S. Barrow

Melbourne Dental School, University of Melbourne, Melbourne, Vic., Australia

Running title: Clinical supervisors' reflections on students' cultural competency

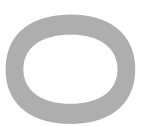

Correspondence to:

A/Prof Rodrigo C: Oral Health Cooperative Research Centre, Melbourne Dental School, The University of Melbourne, Melbourne, Vic. 3010, Australia.

Tel.: +61 (0)393411558

Fax: $+61(0) 393411597$

E-mail: r.marino@unimelb.edu.au 
Keywords: dental students, clinical supervisors, cultural competence; dental education, diversity, multicultural, Australia

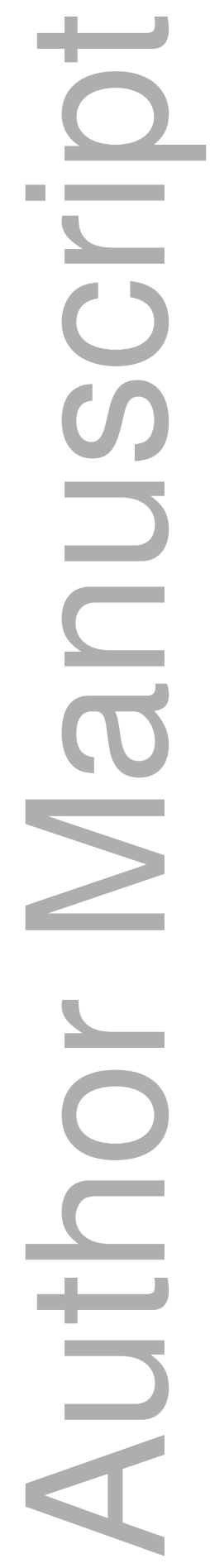

This study explored clinical supervisor's (CS) views and experiences of dental students' cultural competence (CC) at the Melbourne Dental School, The University of Melbourne, Australia. Additionally, this study explored CS insights into how CC could be taught. Methods: Semi-structured one-to-one interviews were organised with consenting CS. 
Interview topics included: the importance of $\mathrm{CC}$, communication and rapport, the role of culture in oral health and the need for curriculum enhancement. Interviews were recorded, transcribed and thematically analysed to identify key areas using NVivo software. Results: A total of 12 CS participated in this study. CS acknowledged the importance of CC and felt that it was important for good patient management. CS's definition of CC focused primarily on language and communication skills. CS felt that dental students were generally able to manage culturally diverse patients. However, CS indicated that additional training in this area would be beneficial. Concerns were raised about the students' ability to establish good rapport and communication, with CS highlighting areas such as misuse of interpreters and use of jargon. CS felt that clinical experience, confidence and a positive attitude are effective tools for overcoming cultural barriers. Furthermore, some CS also felt that cultural competency was a skill that is learnt through experience. Conclusions: For most CS cultural competence was an important part of the clinician-patient exchange which would benefit from enhanced curriculum. They also highlighted areas where transcultural education could be improved. The majority of CS believed dental students managed culturally diverse patients well.

\section{Introduction}

With the increase in immigration and multiculturalism in most societies, the issue of how cultural differences influence community live, education, healthcare, business and trade, and any situation that requires management of diversity, has been an area of growing interest and challenge (1-3). Concerns have also been raised in the literature about immigrant populations receiving inferior healthcare due to communication issues (4-7). In this context, the field of 'cultural competence' has emerged and has become an increasingly important strategy for reducing health disparities $(1,8)$. Furthermore, in a global community, cultural competence may determine an organizations' success (3).

Cultural competence may be defined as a process in which an organization and an individual (e.g., healthcare providers) have a continuous commitment to learn about cultural differences and develop skills to understand the cultural dimensions of a health interaction and work effectively within the cultural context of a client to achieve similar outcomes across culturalethnic groups (2,9). Principal areas of cultural competency (CC) outlined in the literature include the following four components: (a) Awareness, (b) Attitude, (c) Knowledge, and (d) Skills, which must be considered together in CC education (2). This highlights the need for self-reflection, empathy, curiosity and respect $(9,10)$, as well as understanding the importance 
of the patient's perspective, opinion, beliefs and his/her psychosocial and cultural contexts (11).

Cultural competence skills can be acquired, practised and perfected (10). Deficiencies in CC during health care delivery has led to diagnostic errors, missed opportunities for screenings, and inappropriately prescribed medications (12). CC in health care will contribute to the reduction of health disparities among patients. Methods of tackling the teaching of CC have been researched in the fields of medicine $(11,13,14)$ nursing $(5,15)$, and dentistry $(16-20)$. Despite this, there are reports of a lack of awareness of the importance of cultural competence in dental education (21).

Australia's population is both multicultural and diverse, with migrants derived from over 200 countries and 124 religious groups, speaking close to 400 different languages, when indigenous languages are included $(22,23)$. The rising focus on CC in health care delivery has led educational institutions across Australia to include transcultural education in the training of current and future health professionals (24). In fact, many Australian institutions acknowledge CC as an important and necessary skill for their graduates and are reflecting on the level of cultural competence in their curriculums (24-27). Nonetheless, a study found that the dental curriculum of the Doctor of Dental Surgery (DDS) and Bachelor of Oral Health $(\mathrm{BOH})$ at the Melbourne Dental School, University of Melbourne have limited formal content covering transcultural concepts and skills (e.g., less time devoted to teach these concepts) compared to the Master of Nursing Science course (28). In another study at University of Melbourne Dental School, the majority (72\%) of students and new graduates perceived the coverage of socio-cultural aspects in the course as inadequate (29).

However, while the formal transcultural content within curriculum might be limited, students might be exposed to additional content and opportunities for learning within direct interactions with their clinical supervisors (CS) when treating patients during their clinical placements (28). In this context, interactions within clinical settings would provide opportunities for transcultural competency education. Observation is defined as the gold standard for the assessment of CC (30). CS directly observe the student clinician-patient interaction and would, therefore, be able to give an insight into dental students practicing cultural competence.

Thus, the perspective of the CS would give an understanding of the adequacy of the current dental curriculum's coverage of CC and any situations that commonly occur in the student to patient exchange, is particularly valuable for this assessment. This paper aims to explore CS views and experiences of dental students' cultural competence at the Melbourne Dental 
School, The University of Melbourne, Australia. Additionally, because of the need to assess the role, ability and participation of clinical supervisors in teaching the principles of cultural competencies, this study explored CS insights into how CC could be taught.

\section{Materials and Methods}

With approval from The University of Melbourne, Human Ethics Committee (No. 133953), clinical students' supervisors who had a minimum supervising experience of three years, were recruited. Twenty supervising clinical demonstrators satisfied this criteria and were invited to volunteer in this study by taking part in a face-to-face interview. An interview guide was developed in the planning stage of the study. The guide consisted of nine themes to elicit participants' perspectives on the importance of cultural competence; cultural factors associated with communication and rapport; the role of culture in oral health; and curriculum enhancement in CC. Each theme had additional probes to prompt participants to reflect on more focused content. Interviews were conducted by two researchers who independently completed the task, between May and August 2013. Recruitment continued until data saturation was achieved.

Interviews were audio-taped and transcribed verbatim. The analysis process was undertaken concurrently with data collection so that wherever new themes emerged, they were explored further in subsequent interviews until no new themes were uncovered (i.e. point of saturation). Two researchers independently identified preliminary codes from the interview transcripts as they emerged. Codes were discussed until consensus was reached on appropriate codes and their meanings. Transcribed interviews were entered into NVivo software (QSR International Pty Ltd. Version 10, 2012). Data were organised into relevant codes which were then analysed and grouped into themes to identify and group together issues that arose during the interviews. Consistent with grounded-analysis, the analysis used a systematic approach to review and reorganise the transcriptions for individual accounts suitable for thematic analysis; extract 'key ideas' by means of an open-coding method to identify the properties and dimensions of emerging concepts; and identify patterns and themes to provide a map of the concerns and beliefs of the participants $(31,32)$.

\section{Results}

Twelve clinical supervisors, with an average supervising experience of 7.9 years (range: 3-23 years) participated in the study. When asked about the importance of Cultural Competence, the majority felt that cultural factors were important for good patient management. However, even those who felt that cultural factors were less important, conceded that cultural awareness can improve communication and rapport-building between the student and patient, and that 
students who were able to understand the patient's culture tended to achieve better rapport. In fact, most of the CS said that they regularly gave advice to students regarding cultural sensitivity and patient management. As expected, the reported need and frequency of this advice was greater in students in their first clinical year, and decreased as students approached the final clinical years.

CS felt that students were, on the whole, able to adequately manage the student-patient clinical interface and provide treatment to patients from different cultural backgrounds, as reflected by the reduced number of patients' complaints. Despite this, CS discussed several issues which would affect: all students with all patients; and all students when treating culturally and linguistically diverse (CALD) patients. They also highlighted issues that only affect CALD students with all patients. Nonetheless, within this framework, CS noted that when there was a problem with a patient, sometimes it was difficult to know whether it was caused by the student or patient's culture, or just the student's lack of experience.

Factors affecting all students and all patients:

When discussing issues that affect all students and all patients, CS described situations around rapport building as the most relevant. For them, good rapport was the basis upon which a practitioner could get information, manage the health problem, and build and incorporate additional messages to the patient. For some CS, cultural background was less relevant to rapport building, but more related to communication skills. CS expressed the wish to see the student more engaged with the patient, building stronger rapport and gaining more experience in how to treat patients as individuals. Indicating that, patients' management and rapport building, are areas needing curriculum enhancement.

They felt that dental students tended to focus on completing the treatment rather than building a strong rapport with patients. CS commented that although the treatment might have been conducted properly, sometimes they wondered whether communications actually happened.

"They are so pre-occupied with getting the treatment done... it doesn't leave them much space for the subtleties of human interaction."

According to CS, this would be for several reasons. Lack of rapport building was attributed to: students not being graded for their cultural or communications skills, but mostly for their technical skills in completing procedures and treatments; students using dental jargon when talking with patients (e.g., 'caries' or 'restorations'), without recognising that the patient may have not understood (The inappropriate use of dental jargon was seen more in students who 
were inexperienced and/or with poor English language proficiency); students' inability to tailor explanations to the patient's level of education and health awareness.

"I had always assumed that lack of receptiveness was due to communication breakdown with the student...the spiel they give is a one size fits all, they still haven't learnt to... reduce the amount of information they give."

CS provided several ways to establish a good rapport with patients (See Table 1). Regular and rigorous evaluation was seen as essential to meet objectives in communication skills and cultural competence.

"I guess that the risk - there's always a risk - is that trainings, like cultural training, is not something that you going to be tested on."

In fact, CS also attributed patient behaviours to 'personality factors', rather than cultural issues and stated that the student's and the patient's personalities were a more important consideration than their cultural backgrounds. A mismatch between patient and student could potentially result in friction as the patient may perceive the student as lacking confidence or being overly aggressive.

"It's hard to know if they were specific cultural issues or if they were just a simple personality, hum, misunderstanding, or even if it was just the student was particularly young, so just didn't understand, or if the patient was particularly hard to get along with for some reason."

It was suggested that students' confidence and attitudes had a greater influence overall in establishing good rapport, rather than a common cultural background. Lack of confidence might cause an inability to take control of the situation. Dental students are sometimes too deferential to the CS, which the patient may misconstrue as a lack of confidence, thereby diminishing patient's confidence in the student. Students are trying to get clinical experience, which in itself provides more confidence, to deal with patients. In contrast, a few CS noted that some students tended to 'talk down' to their patients.

\section{All students and CALD patients:}

The most obvious clinical situation that would involve all students and CALD patients is the use of interpreters and the issue of cross-cultural communications. In general, CS reported good cross-cultural outcomes:

“They do very well actually. In some instances, I'm quite astounded to see how well they perform because not only are they dealing with somebody whose language is quite foreign to them, obviously, but they are able to break their 
communication down into manageable components for both interpreter and patient, and then carry out the procedure that they're required to do."

Most CS also indicated that rapport was generally poorer when an interpreter was used and suggested several strategies to improve rapport while working with an interpreter. For example, maintaining good eye contact.

"I think what we've already said, you know, maintaining eye contact between the student and the patient, and not just the student and interpreter, is important, and I don't know that students always do quite understand that exactly."

Additionally, students are advised to speak directly to the patient, not to the interpreter. Other issues mentioned included loss of non-verbal cues, longer treatment time, and students were sometimes being unsure when an interpreter was required.

Most CS could recall an instance when the student was unable to manage a patient of a different cultural background and required their intervention. However, all CS were of the opinion that such instances were uncommon and were usually associated with a particular patient.

"Look, I think, by their very nature, the fact that you are not communicating directly with the patient, the fact that you're not getting a sense of their nonverbals, some of the language and intonation, the subtle differences certainly are lost when you use an interpreter, so that can be a barrier."

Barriers mentioned by CS when using interpreters are summarised in Table 2. Perhaps more training is needed in the use of interpreters so developing rapport while an interpreter is present can be practised prior to entering the clinic. CS were not clear whether student got any training on how to work with interpreters, but they acknowledged that learning how to work with interpreters is also self-learning by experience. This comment contrasts with a study on Australian dental students where the majority of students reported feeling fairly or very competent with working with an interpreter (22).

CS indicated that the presence of interpreters could hinder communication, with possible medico-legal implications. For example, informed consent can be more difficult to obtain, and the possibility of not passing relevant information to and from the patient increases.

Furthermore, CS also identified that language and communication problems can also arise even when the student and the patient are both native English speakers, for example, with patients with broad Australian accents.

This article is protected by copyright. All rights reserved 


\section{$\underline{\text { CALD students to all patients }}$}

In particular, communication with patients with broad Australian accents was a challenge for CALD students.

"I have spoken about a couple of students whose English is not very good, whose accent is very strong. And I had to explain to them that you are difficult to understand for the average person - doesn't matter what background they are from... You need to face people, take your mask off, and speak slowly. And ask the patients if they understand, because patients sometimes feel a little bit... they don't want to be rude and sometimes they just go, 'Uh-um...' and they don't really know what you're talking about."

A few CS suggested that students might not have recognised that issues were related to culture, due to lack of awareness.

"They (students) are not really interested in all this background kind of things. They can't see the direct link."

While for some CS, culture has less relevance in their rapport building and more difficulties were seen as SES (socio-economic status) than cultural.

"They are the things that matter, not that I look at you and you're Chinese. The minute you speak good English, anything else, the face doesn't matter, and all the rest doesn't matter."

For other CS, there were real cultural issues that went beyond language, but not communication. For example, incidental conversations, use of colloquialisms, etc. for rapport building may be difficult when there is no common language, but also when there are no common themes (sports, hobbies, etc.), which are important cultural elements, but also useful tools for rapport building (29).

Nonetheless, culture is more than language, communication and rapport. CS mentioned differences between ethno-cultural groups that extend beyond linguistic characteristics. For example, touching, proximity and personal space, being assertive, confrontational, how patients express pain, and the importance of using names when referring to patients, were mentioned as culturally mediated situations.

"Whereas in Asian culture - well, with my culture actually - it's more a mark of respect to be indirect. So if you say their name, it's very rude. In that culture, to say a person's name is very rude, so you would often refer them by their title or indirectly." 


\section{Priorities, expectations and treatment options}

CS also mentioned other clinical situations, which were seen as culturally mediated, with some variation among the different groups. A common point was that patients' expectations played a large role in their management. CS commented on how the patient's culture may affect their expectations, beliefs, and approach to oral health. They highlighted the difficulties of relating to patient's attitudes in terms of differences in motivation, expectations, treatment options and outcomes, which may differ from the clinician's perspective, resulting in conflict.

“... most patients come in with inflated ideas about implants and crowns and bridges and they want all this wonderful treatment that is just not really on the table and the students don't know how to deflect, they don't know how to push off and they don't know how to say no, but that is across all cultures it is a student thing and an inexperience thing rather than a cultural issue as such."

Nonetheless, CS considered that established groups were more familiar with the way the public oral health care service operates in Australia, suggesting that acculturation might have a role. Recent migrants were identified as having increasing demands on public dental services, which may create some difficulties, but this was seen as due to misinformation before and/or after arriving in Australia, than culture. For example, misinformation on the type of oral health care provided in public clinics, confusion over health care cards entitlements, ete. requesting complex treatment (e.g., crowns or implants), or problems with the amount of time taken for treatment.

CS also thought that those were situations where culture had no role in creating expectations. They noted that patients, across all cultural groups, who had been well-advised on what to expect during their visit, were generally satisfied. This is very important as it would reduce misunderstanding and possibly increase the perception of clinical competence amongst all patient groups, including both recent and established migrant groups, as well as AngloAustralian patients. Treatment is limited to the student's scope of experience and training, which results in longer treatment times and precludes the provision of complex procedures by the student. Also, students are required to perform a thorough history, examination and treatment plan prior to treatment. This may not be well understood by patients.

"I think that once they have accepted the fact that they are going to be seen by a student, they tend to be very receptive to that, but as I said some of the patients aren't well vetted and as a result they will expect more than they can 
give and I think that is across cultural boundaries rather than a particular culture expecting too much."

CS commented that cultural beliefs and expectations were also seen as potentially influencing treatment choices regarding dental treatment. For example, unnecessary tooth extractions, shaving of teeth, gold restorations, etc., have cultural meanings. Nonetheless, these issues were considered rare.

"In (XX) culture, it's common to have the lower anteriors (incisor) out, it's aesthetic to have a gap between the $3 s$ (canines)...there are (XX) who like to have one of the upper anteriors (incisor) extracted."

\section{$\underline{\text { Cultural issues }}$}

CS also noticed different cultures as having diverse priorities, perceptions or attitudes regarding oral disease and oral hygiene practices; gender issues; and cultural events. For example, according to CS, newly arrived immigrants seemed less concerned about maintaining good oral hygiene. Nevertheless, CS understood that their circumstances may have mediated on this prioritisation, as under some circumstances (e.g., living in refugee camps), the importance of health and oral health, in particular, might be overshadowed by other priorities (31).

"Newly arrived...probably less concerned. And then when you've got the change of diet, change of habit, you know the whole cultural shift, that can throw everything into disarray, so oral hygiene is probably the last thing on the parent's agenda."

Ramadan was a well-recognised event, mentioned in almost all interviews. Most CS said it was usually not a major issue among the students and that CS tended to remind students that it was approaching. Some CS identified gender issues as a challenge, mostly with Muslim patients refusing treatment from a male clinician. Once it was explained that they were randomly assigned, some patients were understanding and accepted care from a clinician of a different gender. Otherwise, the issue was overcome by reassigning the patients to another student or demonstrator of their gender preference.

"Muslim women who come with their husband and no man is allowed anywhere near the bay, which makes it very difficult. When they've been allocated to a male dental nurse or a male demonstrator, that's not easy." 
Oral hygiene was another area mentioned by CS where patient's needs may not coincide with students viewpoints about proper oral hygiene. Nonetheless, these elements can be incorporated in a treatment plan that is sound, reflecting and respecting patients' values.

"I don't know if there is preparation before the students get to clinic for that.

Because if there is a situation like when a patient uses a chew stick, then the student might not know how to incorporate that into the treatment plan, into the oral hygiene, we don't tell the patient 'oh no, no, that's no good, use a soft toothbrush'. I don't know that students really know how to handle that, they might be a bit confronted and don't know how to deal with it, so they might just dismiss it."

\section{Curriculum Enhancement in Cultural Competency}

CS were asked whether they felt changes in the curriculum were necessary to improve the CC of dental students. The perceived importance was highly variable, paralleling the perceived importance of culture. CS acknowledged that the current curriculum and examinations had little focus on cultural issues. For some CS, teaching the cognitive base of CC was seen as not difficult. For them, formal teaching (e.g., lectures) helps with fundamentals, but the establishment of an environment where the process of achieving CC takes place was perceived as more difficult and harder.

"I could certainly see the value in it, certainly before the student starts clinic. It's a broad topic, and most of the lessons are the ones that are learnt through experience, but certainly some sort of lecture would really help with fundamentals, and especially now that it occurs to me, all the different backgrounds that the student comes from, yeah, a lot of students don't understand."

They considered that CC should remain opportunistic and centred on the clinical experience. In fact, CS questioned whether CC could be addressed through the formal curricula at all.

"Curriculum enhancement is not really necessary because a lot of it is just experience, and not just experience in treating the patients, but also understanding their background and their community".

CS particularly pointed out that learning about CC should not occur solely in the clinical years, suggesting that it should be a theme across the curriculum, permeating the entire learning experience. Furthermore, one CS commented that it is a skill that would help you in your life as a whole:

This article is protected by copyright. All rights reserved 
“That's a life skill, you know. It's not just going to help you in your dentistry, it's going to help you in your whole life."

Types of training suggested by CS to supplement direct clinical experience, ranged from didactics (lectures) to interactive lessons (problem-based learning or role play).

"Problem-based learning, like small groups where you can actually interact;

and maybe even practice, you know, role play."

The majority of CS had the opinion that cultural exposure and clinical experience via real-life or simulated situations that challenged students' concepts and understandings of managing culturally diverse patients were the most effective methods. That is, it should occur both as explicit (coaching) and as implicit learning in the clinic, as part of a "hidden" and informal curriculum.

CS also discussed their role in CC education. They suggested that students learned patient management skills by modelling their CS or other students; hence it may be useful to train CS in $\mathrm{CC}$ as half of the CS interviewed admitted that they may not have had adequate training. As a minority of CS felt that cultural factors were not important in the clinical setting, it is possible that they could unconsciously be modelling this to their students, inhibiting good patient management.

"The students...they tend to follow their demonstrator. They are modelling the way their demonstrator deals with different patients."

\section{Discussion}

The presence of a sizable CALD population poses specific challenges to the various social and health services of a country. Patients from different cultures may have specific expectation and cultural representations which differ from that of mainstream patients (e.g., AngloAustralians). These contextual issues must be recognised and dealt with by oral health professionals using self-reflection, empathy, caring and respect. In the present study, there were a number of cultural issues raised by CS that students had to both understand and overcome for good patient management. However, the oral health needs of patients from CALD backgrounds are not different from those of any other community. Therefore, listening, self-awareness, negotiation and adaptation were seen as essential skills for patient-centred care. At a basic level, all patient-dentist encounters are intercultural, as each one brings with it patient and dentist world views and perspectives $(33,35,36)$. Cultural contacts are rarely straightforward (36). However, negotiating with patients and identifying available information from the patient's perspective can help to minimise conflict and establish rapport (36). These 
skills can be learned and used as means to improve students' cultural competence, so that, when facing cultural differences, students with increased CC can accurately perceive and decode relevant information and adapt behaviours and approaches accordingly (3). Furthermore, their continued use will increase students' self-confidence in their own ability to solve cultural differences.

CS viewed language as the way to describe culture and diversity. Culture was seen more as one component of a whole, than a unifying element that contains language and communication, world views and representations. Restricting culture to country of birth, or language spoken only obscures the possible ways in which culture may operate. A common language is the basis for effective communication. However, communication breakdown may occur, not as a result of word differentiation, but because the language represents a different structure of reasoning, world views, value orientations and belief systems (36-38).

As described by CS, interpersonal communications also involves non-verbal communication, rules and conventions, and other acceptable behaviours associated with communication $(3,36)$. This is important as effective interpersonal communication increases patient satisfaction and compliance, reducing patient anxiety and the risk of malpractice claims (39). Furthermore, a culturally competent practice provides patient-centred care, which has been linked to better clinical outcomes and increased productivity $(27,36)$. These are aspects that the students are very concerned with, due to their clinical assessments.

Cultural competence was identified by $\mathrm{CS}$ as an important part of the student-patient relationship. While most CS reported a good level of CC among students, lack of complaints is not a good marker of insensitivity, cultural biases, or lack of CC. In any case, CS believed that students would benefit from additional cultural training. They raised the need for improvement in cultural education, with incremental emphasis on patient management, CC and communication skills, not purely technical skills, to ensure students are culturally competent in managing an increasingly diverse patient population as they progress in their professional careers. In particular, CS stressed the need for more resources to adequately prepare students to treat CALD patients in dental public clinics.

No doubt there have been improvements in the last few years in the teaching of cultural competence at MDS. However, a review revealed limited cultural competence and crosscultural communication content in the dental curriculum $(25,28)$. In contrast, when dental students were asked about treating CALD patients, they reported feeling competent in communicating effectively, respecting cultural beliefs, working with an interpreter, 
establishing rapport and communicating diagnoses (29). Furthermore, over $70 \%$ of students reported that they rarely or never encountered patient's cultural beliefs which could interfere with diagnosis and treatment, but felt less skilled in dealing with cultural practices (29). However, student may not have the knowledge or experience to identify differences that might influence a clinical encounter (40).

Issues surrounding the use of interpreters have been identified in the literature $(6,7,36,41)$, including health practitioners spending less time talking to patients when an interpreter is present, less information being conveyed due to fixed consult time and the amount of time spent on translation $(36,41)$, as well as a lack of training to work with interpreters $(6,41)$ and concerns about the precision of translation (6). These were echoed by participating CS who suggested that interpreting, although necessary, was time-consuming and hindered communication.

There have been a number of studies on the ability of oral health practitioners and students to adapt to treating patients of different cultures (29,33,42-45). Most of these studies addressed cultural differences and the teaching of CC, and were conducted in the United States $(16,18$ $20,33,42,43,46-48)$. Studies that investigate CC teaching in oral health professional programs in Australia are needed to ensure that it becomes a skill of every practicing oral health professional.

There is an extensive body of literature on the role of education to improve communication skills and CC among health professionals $(30,49)$. However, there is no magic formula to ensure that the unique cultural needs of every patient are addressed. In line with the literature, the main approaches cited by CS to facilitate the learning of the cognitive basis of cultural competence included a variety of teaching exercises that enable feedback on course content and student response $(17,19,45,49)$, practical exercises [role playing and simulation] $(3,16,20)$, clinical rotations in culturally diverse communities $(19,21,49)$ and self-evaluation [reflective diaries about personal opinions concerning treating culturally diverse patients and their CC training] $(17,33,21,49)$, as well as interdisciplinary courses with other health practitioners (19). It was also suggested that increasing the proportion of students from ethnic minorities within dental schools, and exposure to as wide as possible a variety of cultures may help address challenges associated with cultural diversity and cultural learning (2,3,46,47). Yet, while these students may be bilingual, they may not necessarily be bicultural as even when the students' backgrounds were "diverse", there was a common cultural profile to which all students subscribed (44). These discussions highlight the complexities of a culturally competent learning practice.

This article is protected by copyright. All rights reserved 
As with any study, this one is not without limitations. The most obvious one is that it only represents the views and experiences of CS from a single dental school, which may not reflect the diversity of views in clinical settings of other Australian dental schools. Additionally, only CS motivated to participate were interviewed. Despite these limitations, the study has provided an in-depth exploration into supervising clinicians' perceptions of CC of dental students and cross-cultural issues within the Australian clinical context.

As long as a school continues to produce professionals, researchers, and educators who are committed to cultural diversity, the model for how CC and communication skills are taught and evaluated will require continuous reviews and reforms. Further research is needed to investigate the themes that emerged with a wider sample of supervising dentists from other dental schools. In addition, a future area of study would be to explore cross-cultural interactions from the unique perspectives of dental students and patients. The information gained from such studies would contribute to a more comprehensive understanding of cultural issues in clinical settings, which would in turn serve as the foundation for the development of effective $\mathrm{CC}$ training standards that dental schools are providing to new dental practitioners.

\section{Acknowledgements}

The authors would like to acknowledge the cooperation of clinical supervisors for their participation in the conduct of the study. The authors have no conflicts of interest.

\section{References}

1. National Health and Medical Research Council. Cultural competency in health: A guide for policy, partnership and participation. Canberra: Australian Government, 2005. Available from: http://www.nhmrc.gov.au/_files_nhmrc/publications/attachments/hp19.pdf. (accessed 22 January 2016).

2. Martin M, Vaughn B. Cultural competence: the nuts \& bolts of diversity \& inclusion. Strategic diversity \& inclusion management. 2007;1:31-36. Available from: www.dtui.com/Magazine/MagArticles/March2007pub/cultural_competence.doc. (accessed 6 May 2016).

3. Groves K, Feyerherm A, Gu M. Examining Cultural Intelligence and Cross-Cultural Negotiation Effectiveness. J Manag Educ 2015;39:209-243. 
4. Migrant-Friendly Hospitals. Amsterdam Declaration. Towards Migrant-Friendly Hospitals in an ethno-culturally diverse Europe. Available from: $\underline{\text { http://www.mfh- }}$ eu.net/public/european_recommendations.htm. 2004. (accessed 22 January 2016).

5. Jirwe M, Gerrish K, Emami A. Student nurses' experiences of communication in crosscultural care encounters. Scand J Caring Sci 2010:24:436-444.

6. Kai J, Beavan J, Faull C. Challenges of mediated communication, disclosure and patient autonomy in cross-cultural cancer care. Br J Cancer 2011:105:918-924.

7. Schouten BC, Meeuwesen L. Cultural differences in medical communication: A review of the literature. Patient Educ Couns 2006:64:21-34.

8. Betancourt J, Green A, Carrillo E, Ananeh-Firempong O. Defining cultural competence: a practical framework for addressing racial/ ethnic disparities in health and health care. Public Health Rep 2003: 118: 293-302.

9. Campinha-Bacote J. The process of cultural competence in the delivery of healthcare services: A model of care. J Transcult Nurs 2002:13:181-184.

10. Kodjo C. Cultural competence in clinician communication. Pediatr Rev 2009:30:57-63.

11. Crosson JC, Deng W, Brazeau C, Boyd L, Soto-Greene M. Evaluating the effect of cultural competency training on medical student attitudes. Family Med 2004:36:199-203.

12. Brach C, Fraserirector I. Can cultural competency reduce racial and ethnic health disparities? A review and conceptual model. Med Care Res Rev 2000:57: 181-217.

13. Griswold K, Zayas LE, Kernan JB, Wagner CM. Cultural awareness through medical student and refugee patient encounters. J Immigr Minor Health 2007:9:55-60.

14. Rosen J, Spatz ES, Gaaserud AM, Abramovitch H, Weinreb B, Wenger NS, et al. A new approach to developing cross-cultural communication skills. Med Teach 2004:26:126-132.

15. Murphy ST, Censullo M, Cameron DD, Baigis JA. Improving cross-cultural communication in health professions education. J Nurs Educ 2007:46:367-372.

16. Broder HL, Janal M. Promoting interpersonal skills and cultural sensitivity among dental students. J Dent Educ 2006:70:409-416.

17. Gregorczyk SM, Bailit HL. Assessing the cultural competency of dental students and residents. J Dent Educ 2008:72:1122-1127.

18. Hottel TL, Hardigan PC. Improvement in the interpersonal communication skills of dental students. J Dent Educ 2005:69:281-284.

19. Rowland ML, Bean CY, Casamassimo PS. A snapshot of cultural competency education in US dental schools. J Dent Educ 2006:70:982-990. 
20. Wagner J, Arteaga S, D'Ambrosio J, Hodge C, Ioannidou E, Pfeiffer CA, et al. Dental students' attitudes toward treating diverse patients: Effects of a cross-cultural patient-instructor program. J Dent Educ 2008:72:1128-1134.

21. Al-Shehri H, Al-Taweel S, Ivanoff C. Perceptions of Saudi dental students on cultural competency. Saudi Med J 2016:37:208-211.

22. Australian Bureau of Statistics. 2006 Census of Population and Housing: Media Releases and Fact Sheets. Canberra, ACT: Australian Bureau of Statistics, 2006, 2007. Available from: http://www.abs.gov.au/ausstats/abs@.nsf/7d12b0f6763c78caca. (accessed 22 January 2016).

23. Department of Immigration and Multicultural Affairs. Report of the review of settlements services for migrants and humanitarian entrants 2003. Available from: http://www.immi.gov.au/media/publications/settle/_pdf/chap03web.pdf. (accessed 14 Aug 2015).

24. Mariño R, Hawthorne L, Morgan M, Ismail M. Transcultural skills content in a dental curriculum: a comparative study. Eur J Dent Educ 2010:15:1-7.

25. Mariño R, Morgan M, Hopcraft M. Transcultural dental training: addressing the oral health care needs of people from culturally diverse backgrounds. Community Dent Oral Epidemiol 2012:40:134-140.

26. Hewlett ER, Davidson PL, Nakazono TT, Baumeister SE, Carreon DC, Freed JR. Effect of school environment on dental students' perceptions of cultural competency curricula and preparedness to care for diverse populations. J Dent Educ 2007:71: 810-818.

27. Stewart S. Cultural competence in health care. Diversity Health Institute, Cultural Competence Guidelines and Protocols, Victoria, Sydney; 2006.

28. Mariño R, Morgan M, Barrow S. Transcultural Skills Content in a Dental Curriculum: A Comparative Study. JDR 2014:93(Special Issue B);990 .

29. Mariño R, Morgan M, Hawthorne L, Ismail M. Self-reported cultural skills from dental students and graduates from Melbourne, Australia. Eur J Dent Educ 2013:17:159-165.

30. Sherman JJ, Cramer A. Measurement of changes in empathy during dental school. J Dent Educ. 2005;69:338-345.

31. Corbin J, Strauss A. Grounded theory research: Procedures, canons, and evaluative criteria. Qual Sociol 1990:13:3-21.

32. Strauss A, Corbin J. Basics of qualitative researches: techniques and procedures for developing grounded theory. 2nd edn. Thousand Oaks: Sage Publications, 1998.

33. Victoroff KZ, Williams KA, Lalumandier J. Dental student's reflections on their experiences with a diverse patient population. J Dent Educ 2013;77:982-9. 
34. Nair RG, Samaranayake LP, Philipsen HP, Graham RGB, Itthagarun A. Prevalence of Oral Lesions in a Selected Vietnamese Population. Intl Dental J 1996;46:48-51.

35. Teal CR, Street RL. Critical elements of culturally competent communication in the medical encounter: a review and model. Soc Sci Med 2009;68:533-43.

36. Crisp J,-Taylor C. Culture and Ethnicity. In: Crisp J, Taylor C: Potter \& Perry's Fundamental of Nursing. $2^{\text {nd }}$ Edtion. pp.122-142. Elsevier Australia, 2005.

37. Kluckhohn F. Variations in Value Orientation as a Factor in Education Planning. In: Bower EM, Hollister WG: Behavioral Science Frontiers in Education. pp 293-314. Wiley \& Sons, Inc. 1967.

38. Ibrahim FA. Effective Cross-Cultural Counselling and Psychotherapy: A Framework. Couns Psych 1985; 13:625-638.

39. Yoshida T, Milgrom P, Coldwell S. How do U.S. and Canadian dental schools teach interpersonal communication skills? J Dent Educ 2002:66:1281-1288.

40. Nápoles-Springer AM, Santoyo J, Houston K, Pérez-Stable EJ, Stewart A. Patients' perceptions of cultural factors affecting the quality of their medical encounters. Health Expect 2005;8:4-17.

41. Butow P, Bell M, Goldstein D, et al. Grappling with cultural differences; Communication between oncologists and immigrant cancer patients with and without interpreters. Patient Educ Couns 2011:84:398-405.

42. Finkbeiner BL. Managing cultural differences in the dental office. Dent Assist 2009:78:38-39.

43. Khan FA, Williams SA. Cultural barriers to successful communication during orthodontic care. Community Dent Health 1999:16:256-261.

44. Mariño R, Stuart G, Morgan M, Winning T, Thompson M. Cultural consistency in Australian dental students from two different ethnic backgrounds. J Dent Educ 2004:11:11781184.

45. Tavoc T, Newsom R, DeWald JP. Cross-cultural adaptability of Texas dental hygienists and dental hygiene students: A preliminary study. J Dent Educ 2009:73:563-570.

46. Formicola AJ, Stavisky J, Lewy R. Cultural competency: Dentistry and medicine learning from one another. J Dent Educ 2003:67:869-875.

47. Mitchell DA, Lassiter SL, Lamster IB. Cultural diversity and its role in reducing oral health disparities. N Y State Dent J 2005:71:40-42.

48. Wagner JA, Redford-Badwal D. Dental students' beliefs about culture in patient care: Selfreported knowledge and importance. J Dent Educ 2008:72:571-576. 
49. Rubin RW. Developing cultural competence and social responsibility in preclinical dental students. J Dent Educ 2004:68:460-467.

Table 1. Clinical supervisors' advice to establish a good rapport with patients

1. Be a good listener

2. Explain in lay language/terms

3. Not impersonal. Show empathy, that the patients' health counts; interest in the person/individual.

4. No rushing to get the work done.

5. Use of common language; avoid dental jargon

6. Eye contact

7. Ayoid use of masks when talking

Table 2. Barriers mentioned by clinical supervisors when using interpreters.

1. Non-verbal communication: lack of non-verbal cues (intonations) in the translations

2. Lack of eye contact

3. Decrease rapport building

4. Interpreters physically in the way

5. Extended time required for the treatment 


\section{University Library}

\section{- M M N E R VA A gateway to Melbourne's research publications}

Minerva Access is the Institutional Repository of The University of Melbourne

Author/s:

Marino, R;Ghanim, A;Morgan, M;Barrow, S

Title:

Cultural competency and communication skills of dental students: clinical supervisors' perceptions

Date:

2017-11-01

Citation:

Marino, R., Ghanim, A., Morgan, M. \& Barrow, S. (2017). Cultural competency and communication skills of dental students: clinical supervisors' perceptions. EUROPEAN JOURNAL OF DENTAL EDUCATION, 21 (4), pp.E101-E108. https://doi.org/10.1111/ eje.12227.

Persistent Link:

http://hdl.handle.net/11343/291963 